\title{
Remediation Activities in an Italian Superfund: Case Study of an Industrial Plant in Broni
}

\author{
Sergio Bellagamba, Federica Paglietti, Sergio Malinconico, Beatrice Conestabile Della Staffa, \\ Paolo De Simone, Ivano Lonigro
}

Department of Technological Innovations and Safety of Plants, Products and Anthropic Settlements (DIT) of the Italian Institute for Insurance against Accidents at Work (INAIL)

Piazzale Pastore 6, 00144 - Rome, Italy

s.bellagamba@inail.it f.paglietti@inail.it

\begin{abstract}
The Italian Ministry for the Environment and Protection of the Land and Sea (MATTM) has identified through Ministry Decree n. 468/2001 and its integrations, 40 Italian Superfund within the national borders, 10 of which are contaminated mainly by Asbestos. Within these areas, the INAIL-DIT play a role in finding and managing issues related to Asbestos and Asbestos Containing Materials (ACMs), together with others national institutes. This activity is made by an "ad hoc" working group, with the aim to identify the best Emergency Safety Measures, or remediate these sites, together with occupational and population risks monitoring and prevention, and to ensure the best available technologies. In this paper a very complex site has been described; we explain the remediation project designed after the prescriptions made by the INAIL-DIT experts in order to reduce the contamination in the working area and the living environment.
\end{abstract}

Keywords: Asbestos, Superfund, Remediation, Safety, Asbestos-cement.

\section{Introduction}

The Italian Superfund named Broni Fibronit is placed within the industrial area of Broni, which is a 9.000 people town located in the Province of Pavia. The site has a surface of $140.000 \mathrm{~m}^{2}, 35 \%$ of which occupied by industrial sheds and offices, and the remaining part by squares and internal streets.

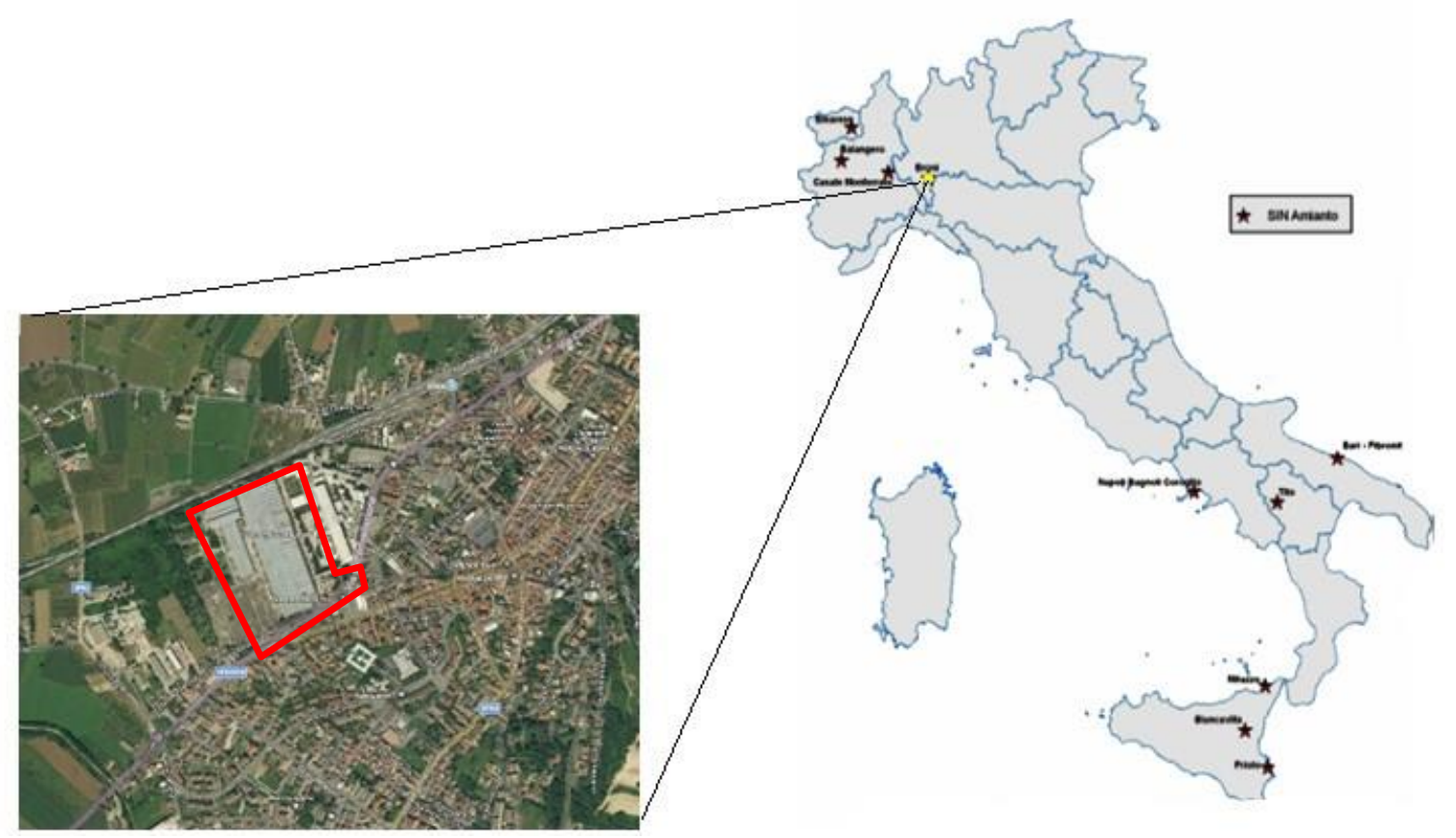

Fig. 1: Localization of Broni Fibronit Superfund. 
In 1932, the plant began the production of asbestos-cement products like pipes, corrugated sheets, flues, special components, fittings, etc. Because of the growing market demand, the Fibronit subsequently upgraded the productive lines to 9, three of which dedicated to the production of pipes, five to corrugated sheets and one to square flues. The plant got to produce more than $10^{5}$ tons/year, employing about 1.300 workers.

The process consisted of the following steps: 1) Material discharge, 2) Mix of various asbestos variety, 3) Mixture preparation, 4) Products shaping, 5) Maturation, 6) Finishing, 7) Final storage. The Fig. 2 shows the pipe's production process, while the Fig. 3 outlines the production of corrugated sheets.

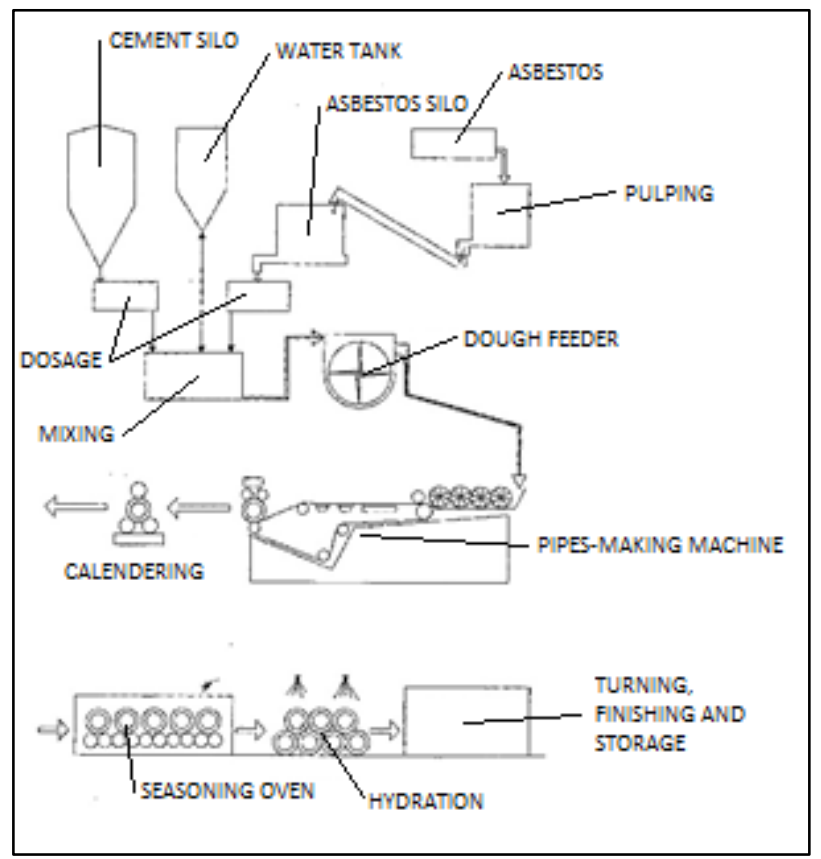

Fig. 2: Pipe's production process.

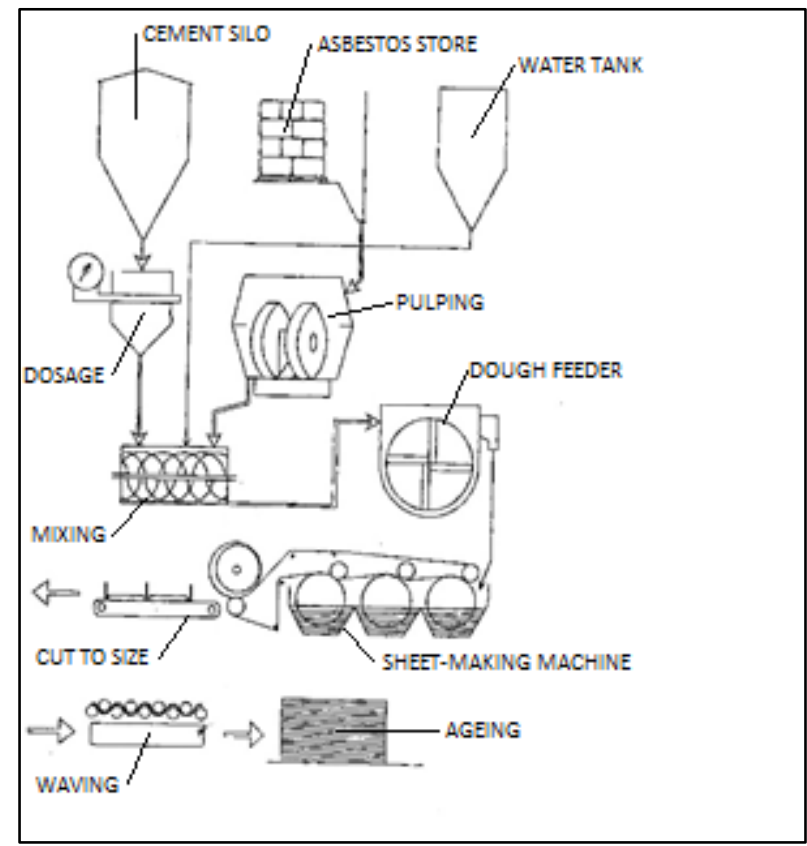

Fig. 3: Corrugated sheet's production process. 
Thanks to the growing knowledge about the hygienic and environmental issues related to the exposition to asbestos fibres, at first the Fibronit implemented an automatic and closed-cycle mixing plant, connected to an HEPA filter battery, so to reduce the dust.

Previously in the mid 70's, the issue about the consequences of the asbestos exposition started to strengthen. Then, the irreversible market crisis related to the scientific evidences on these risks, in Italy finally led to the asbestos banishment, through Law n. 257 of 1992 that established the production's disposal since 1993. Suddenly the plant has been closed and discarded, while the machineries and the materials present during that time have been left in place.

For remediation purposes, the INAIL DIT, during a number of Emergency Safety Measures and remediation activities, delivered many technical-scientific reports of consultancy, with the aim to indicate the principal measures suitable for workers and living environments protection [1][2][3].

\section{Site Description}

After identification by the Ministry for the Environment and Protection of the Land and Sea (MATTM) through Ministerial Decree n. 26 November 2002 and Italian Law n. 179/2002 [4], at present time the site's property is divided by three: the Fibroservice area, the ex-Fibronit area and the ex-Ecored area. Within the last two areas some Emergency Security Measures have been carried out, due to the high potential of pollution spread. In the ex-Fibronit area, all the outdoor Asbestos Containing Waste (ACW) have been encapsulated, packed up and sent for disposal to licensed off-site landfills. All the waste produced during pipes production have been removed from the ex-Ecored uncovered area, which extends for about $450 \mathrm{~m}^{2}$, together with the mud accumulated along the meteoric water channels. In the ex-Fibroservice area, the square has been cleaned up and all the asbestos-cement coverings have been removed. The encapsulation of the asbestos-cement sheets (which formed the cover and the side buffering of the sheds) and the sealing of the openings constituted the Emergency Safety Measures. The Superfund has been divided into remediation lots, as follows (see Fig. 4): the shed B into B1- B6 lots, while shed $\mathrm{C}$ into $\mathrm{C} 1$ and $\mathrm{C} 2$ lots.
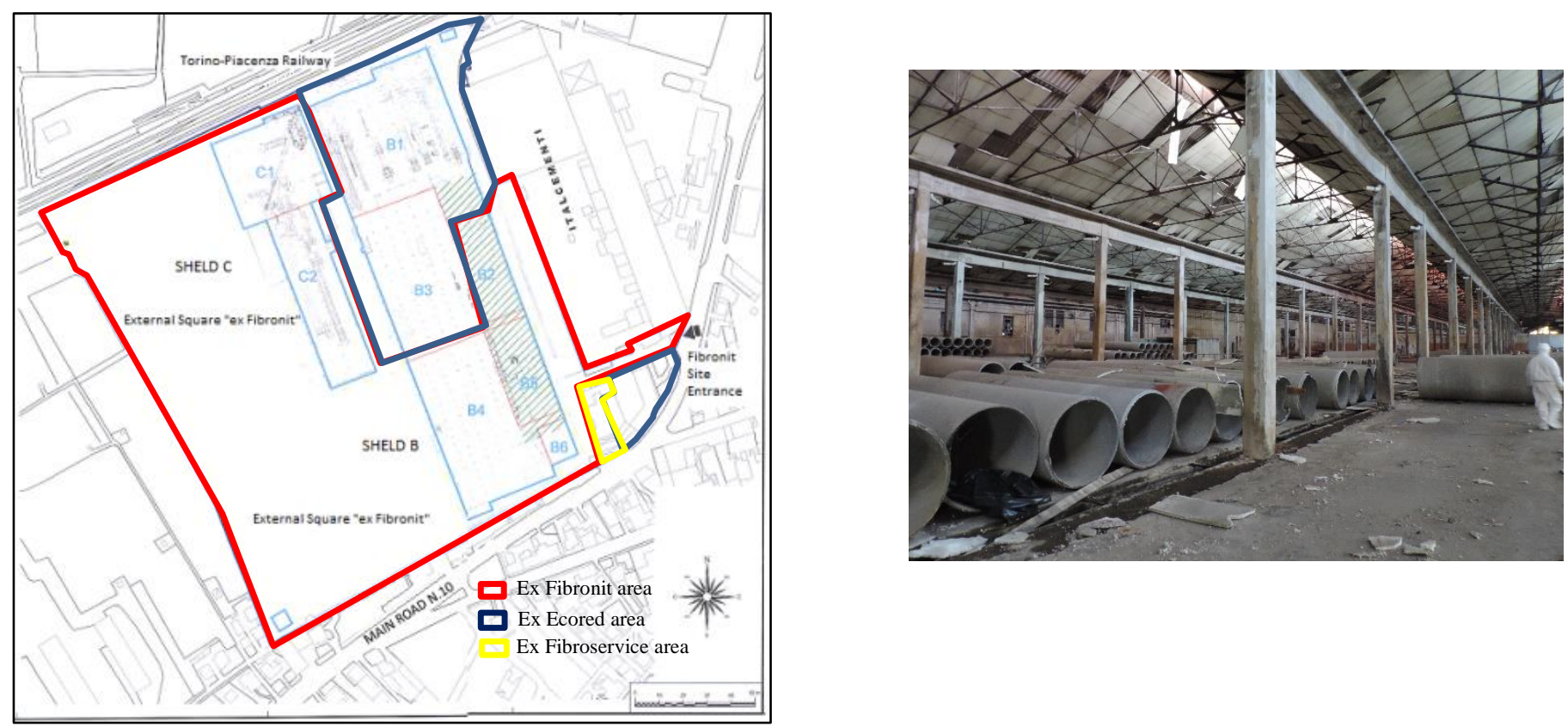

Fig. 4: Map and image of the Broni Fibronit Superfund.

The B1 lot have been completely cleaned up and returned. Actually remediation activities are being carried out within the others remediation lots.

\section{Site Condition}

In 2013 a first remediation project has been presented, regarding the Fibronit property. It originally included:

- The completion of the cleaning activities on B2 - B6 and $\mathrm{C} 1-\mathrm{C} 2$ lots; 
- The removal and disposal of the asbestos-cement sheets from the coverings and the side buffering of the $\mathrm{B}$ and $\mathrm{C}$ sheds;

- Underground tanks remediation;

- Remediation and removal of the silos and the pipes present above the B shed;

- Removal and disposal of the first portion of the underground sewerage system.

The procedures have been subjected to analysis by INAIL-DIT to assess the adequacy with the current regulations and in particular to verify that the activities were carried out ensuring a high and permanent level of safety for persons and the living environment [5]. To do so, the INAIL-DIT has carried out a site inspection during February 2014, in order to verify the site's conditions and the adequacy of the proposed procedures. The site inspection reported some important safety shortcomings for workers and living environments. In particular the site was completely accessible, also due to frequent holes in the fence. There was a lack of signage, both anti-intrusion and danger. There were asbestos containing waste bags, showing no European Waste Catalogue (EWC) classification, uncovered and weather-beaten, with breaking and pollution risks. At the entrance to the offices (all of them accessible and in serious decay), there were founded some asbestos containing powders, to be arranged urgently through Emergency Safety Measures or remediation. Finally, the presence of significant amounts of asbestos cement waste all around the area's internal yards has been highlighted, together with the lack of equipment necessary to avoid the contamination leakage in areas outside the Superfund perimeter. So the INAIL-DIT has reported many prescriptions and the necessity to intervene through the following prior activities:

- Emergency Safety Measures and remediation of the silos;

- Emergency Safety Measures and characterization of some 3.000 tons heap of processing waste;

- Site entrance buildings remediation;

- Static and dynamic confinements of all the building structures before the reclamation activities are carried out;

- Specific procedures for every worker's decontamination activity;

- Removal of all the outdoor waste [6];

- Installation of a suitable fence and signage, both anti-intrusion and danger, and surveillance;

- Set up of Personnel and Materials Decontamination Unit and an incoming/outgoing vehicle washing platform;

- Personnel Protection Equipment (PPE) correct use check (it was noted that many workers didn't use them properly);

- Smoking ban.

Following these requirements, in 2015 the Municipal Authority has presented a new project focused on the Fibronit area and on the following areas, in which the INAIL-DIT prescriptions have been implemented. The remediation activities include interventions within the $\mathrm{B}$ and $\mathrm{C}$ sheds and outside, in the external areas. As already mentioned, the shed B has been partly remediated (B1). Therefore, the 2015 project has envisaged the remediation of the remaining volumes, characterized by heights up to 13 meters, which are reported below.

Table 1: B Shed. Lot's Volumes and Surfaces.

\begin{tabular}{|c|r|r|r|}
\hline Lot & Volume $\left.\mathbf{( m}^{\mathbf{2}}\right)$ & Surface on plan $\left.\mathbf{( m}^{\mathbf{2}}\right)$ & Total surface $\left.\mathbf{( m}^{\mathbf{2}}\right)$ \\
\hline B2 & 41.000 & 3.150 & 15.800 \\
\hline B3 & 93.500 & 9.200 & 22.400 \\
\hline B4 & 107.900 & 10.900 & 26.750 \\
\hline B5 & 44.000 & 3.500 & 23.500 \\
\hline B6 & 6.000 & 860 & 2.550 \\
\hline Total & $\mathbf{2 9 3 . 0 0 0}$ & $\mathbf{2 7 . 6 1 0}$ & $\mathbf{9 1 . 0 0 0}$ \\
\hline
\end{tabular}

Some insulation panels, which at first were fixed to the metal structures of the shed's attics, now are detached and/or fallen to the ground. Inside the sheds, there is a widespread asbestos contamination, due to heaps on the floor and the walls, and because of machinery and production lines encrusted by the polluted material. Furthermore, also the offices of the B5 lot must be remediated, because of a kind of secondary contamination, caused by the high dust levels. Finally, there are some tanks in which the liquid waste was collected for decantation, with total surface and volume estimated as $750 \mathrm{~m}^{2}$ and $560 \mathrm{~m}^{3}$. 
The secondary facility is the $\mathrm{C}$ shed. It's made up of two lot. The $\mathrm{C} 1$ lot is square, the $\mathrm{C} 2$ is narrow and stretched, both characterized by heights up to 13 meters. The following Table n. 2 shows the lot's volumes and surfaces.

Table 2: C Shed. Lot's Volumes and Surfaces.

\begin{tabular}{|c|r|r|r|}
\hline Lot & Volume $\left(\mathbf{m}^{\mathbf{3}}\right)$ & Surface on plan $\left(\mathbf{m}^{\mathbf{2}}\right)$ & Total surface $\left(\mathbf{m}^{\mathbf{2}}\right)$ \\
\hline $\mathrm{C} 1$ & 66.000 & 6.000 & 15.500 \\
\hline $\mathrm{C} 2$ & 42.000 & 6.000 & 14.700 \\
\hline Total & $\mathbf{1 0 8 . 0 0 0}$ & $\mathbf{1 2 . 0 0 0}$ & $\mathbf{3 0 . 2 0 0}$ \\
\hline
\end{tabular}

The shed's roofs and part of its side buffering are made of asbestos-cement sheets. Inside the C1 and C2 lots, there are some machineries, silos, wreckages and processing residues, all encrusted by ACMs. Particularly, inside the heavily degraded $\mathrm{C} 1$ lot, there's a corrugated sheets production machine; furthermore, drying/cooking structures are installed for the entire length of the shed. There is also a $2.250 \mathrm{~m}^{3}$ tank inside the basement.

Finally, a contaminated sewerage system is situated under the B and C sheds and the squares. It has got a total linear development of $3.250 \mathrm{~m}$. Pipes and wells on the bottom are characterized by large muddy deposits (about $20 \mathrm{~cm}$ ) with high concentrations of asbestos fibres. The remediation activities on these underground structures are not included in the project proposed in 2015 and will be subject to future interventions. The remediation project concerns the sheds, the basements and the tanks present within the $\mathrm{B}$ and $\mathrm{C}$ areas. Table 3 shows the surfaces covered by the intervention.

Table 3: Surfaces involved in the remediation.

\begin{tabular}{|l|r|}
\hline \multicolumn{1}{|c|}{ Unit } & Surface to be remediated $\left(\mathbf{m}^{\mathbf{2}}\right)$ \\
\hline B shed & 91.000 \\
\hline B1 basement & 675 \\
\hline B1 Underground tanks & 750 \\
\hline C shed & 30.200 \\
\hline C tanks and basement & 1.940 \\
\hline Total & $\mathbf{1 2 4 . 5 6 5}$ \\
\hline
\end{tabular}

In the present paper we explain the steps which present the highest impact on worker's safety and the living environments. The principal steps are: A) Preparation of confinements, B) Sheds emptying and remediation, C) Removal and disposal of asbestos-cement sheets, D) Remediation of underground tanks, E) Restorability of asbestos-remediated areas. The related procedural details are shown below, because these methods can be taken as example for similar national or international situations, in other disused ACMs production plants.

\section{Analysis of the Remediation Project with Implementation of the INAIL-DIT Requirements 4.1. Preparation of Confinements}

The original project included the preparation of a confinement every $30-40.000 \mathrm{~m}^{3}$, through creation of about 17 static and dynamic confinements. The INAIL-DIT has requested to reduce the volume of every confinement up to $10.000 \mathrm{~m}^{3}$, which make it more functional and easy to prepare. The volumes are considered suitable after a correct smoke test made to ensure the saturation of the working area and check if there are any flaws on the outside that make smoke escape. Any leaks must be sealed inside. Currently about 36 confined rooms of variable volumes have been designed, whose sizes are shown in Table 4. 
Table 4: Confined areas dimensions. *One or more raised floor presence.

\begin{tabular}{|l|r|r|r|}
\hline \multicolumn{1}{|c|}{ Unit } & $\begin{array}{c}\text { N. of confined } \\
\text { areas }\end{array}$ & $\begin{array}{c}\text { Single confined areas } \\
\text { volumes }\left(\mathbf{m}^{\mathbf{3}}\right)\end{array}$ & $\begin{array}{c}\text { Surface } \\
\left(\mathbf{m}^{\mathbf{3}}\right)\end{array}$ \\
\hline B2 Lot & 4 & 10.250 & $6.300^{*}$ \\
\hline B3 Lot & 8 & 11.687 & 9.200 \\
\hline B4 Lot & 10 & 10.750 & 10.900 \\
\hline B5 Lot & 3 & 14.666 & $10.000^{*}$ \\
\hline B6 Lot & 1 & 6.000 & 860 \\
\hline C1 Lot & 6 & 11.000 & 6.000 \\
\hline C2 Lot & 2 & 21.000 & 6.000 \\
\hline B1 Basement & 1 & ---- & 250 \\
\hline B1 Underground tank & 1 & ---- & 280 \\
\hline Total & $\mathbf{3 6}$ & & \\
\hline
\end{tabular}

Each confinement system will be equipped with an air extraction system consisting of enough extractors to ensure an internal depression gradient. Systems, equipped with HEPA filters 99,97\%, must reduce the concentration of airborne asbestos fibres inside the working area and promote an optimal air exchange for the improvement of working conditions. Finally, these plants must be kept in operation 24 hours a day, from activation to final decontamination. The shutdown will be authorized by the local control Authority, only after verification of contamination absence and release of the restoration certificate.

\subsection{Sheds Emptying and Remediation}

The original project provided the collection of all ACMs and those without asbestos, deposited on the ground and on machineries, a floor cleaning by aspiration system and storage of the ACW inside a not delimited site storage area. For the cumbersome materials and structures it was provided to decontaminate and leave them on the ground in the remediated buildings with possible dismantling only after the release of the restoration certificate.

Following the transposition of the INAIL-DIT provisions, the new project provided that the materials must be encapsulated, place in closed bags and send to the Materials Decontamination Unit (MDU), where they must be re-packed in special big-bags.

These types of materials classified as ACW (in friable matrix and in compact matrix) must be stack for similar categories and distinct by EWC code in a designated temporary static and dynamic confinement and equipped with appropriate signage and access allowed only to the workers.

The waste no containing asbestos but only contaminated by asbestos must be remediated and stacked at a further confined area, distinct from the previous one. Afterwards, in this area, the waste must be checked at regular intervals by local control Authority to verify the total absence of residual contamination.

It also provides, in the first phase of shed's emptying, to disassemble and remove all the machineries and structures contained inside the sheds, using a static and dynamic confinement [7].

Also the silo must be brought to the ground, subsequently emptied and dismantled inside a static and dynamic confinement and then disposed. Given the high level of contamination of the structures and machineries to be remediated, the MDU must be composed of 4 distinct areas (only 3 are required by Italian Law - Ministry Decree n. 6 September 1994): this in order to guarantee a greater resilience of confinement and a better degree of outgoing materials decontamination. Inside the first area of the MDU (pre-wash area), a special prewash tank with water must be put, so to completely immerse and impregnate the metal waste and allow the deposition of surface contamination.

In the second area the metallic waste must be wash with a low pressure nozzle and at the same time it must be brush and descale; all these operations must be done by hand on the waste. Afterwards, the waste must be deposited in the third area and must be stacked in separate lots of the same size, so to ensure their inspection by the local control Authority. In this area 
the samples must be taken for subsequent analysis by the designate laboratory and related re-test carried out by the local control Authority (over $10 \%$ of all samples taken).

The waste can be transferred to the fourth area, only after of the decontamination certification released by the local control Authority. There, it can be taken from outside by a team of non-contaminated workers and sent to the temporary storage area.

Regarding the structures decontamination procedures, INAIL-DIT required that the descaling operations, decontamination and final cleaning must be carried out proceeding from top to bottom starting with an aspiration of the superior metal structures and the removal of the suspended ceilings; subsequently proceeding with the decontamination of the side panels from various encrustations through the use of manual tools and water.

The ACW must be encapsulated, put in sealed bags and transported in the MDU. After the last operation the whole indoor area must be washed and encapsulated, starting from the top to the flooring, so to gain the restoration certificate from the local control Authority regarding all the working area.

\subsection{Removal and Disposal of Asbestos-Cement Sheets}

In the first project the asbestos cement sheets removal was designed without indicating specific operating procedures. The INAIL-DIT, however, has requested that the asbestos-cement sheets located inside the B and C sheds (with a total surface of approximately $68.000 \mathrm{~m}^{2}$, corresponding to about 1.230 tons), must be the subject of a preliminary suction of the surfaces and dust accumulations or fragments - manual - removal, through wetting and subsequent bagging. The next step is the permanent encapsulation through vinyl with an average thickness of 300 microns, to be carried out using a low pressure pump. The sheets must be removed intact, avoiding demolition and using manual tools; any inconsistent materials (dust, sludge, garbage, etc.) showing edges and pieces must be packaged separately from the sheets inside rigid containers and disposed in hazardous waste landfill, with the EWC code 10.12.09* [8]. The sheets, dismantled one by one and encapsulated on both surfaces, must be suitably packaged, labelled with the asbestos danger symbol and specific indication of the EWC code $17.06 .05^{*}$. Then, by the end of the working day, the ACW must be put in temporary storage with static and dynamic confinement, located on site. Subsequently, at a reasonable volume with transport on vehicles, it must be sent to landfill.

\subsection{Remediation of Underground Tanks}

In the original project was proposed to empty the underground tanks and to accumulate the water in a cistern and then characterize it. On the other hand, INAIL-DIT requested that the tanks should be emptied by pumps and the water filtered through a special system or disposed with EWC code 16.10.01* - aqueous waste solutions containing dangerous substance. Considering the probable asbestos contamination, the tanks must be remediated inside a statically and dynamically confinement with relative PDS and MDS, to be tested through smoke test with the presence of local control Authority. Therefore the tanks remediation will include the removal of muddy deposits from the bottom and its disposal with the EWC code 19.13.03*.

\subsection{Restorability of Asbestos-Remediated Areas}

According to the Italian law, at the end of the asbestos remediation activities, the local control Authority carries out visual inspection of the remediated areas to check for the presence of residues of ACMs or powders containing asbestos; at the same time the sampling of deposited powders and an airborne particulate sampling are required, followed by analysis through scanning electron microscope [9]. The verification of the concentration of the airborne asbestos fibres must be carried out through aggressive samplings, to be carried out using industrial fans designed to create a suspension of the eventually deposited powders. The analysis of the airborne particulate must demonstrate that inside the sheds there is a concentration lower than 2 fibres/l (reference limit value). Only in these conditions the statically and dynamically confinement restoration certificate can be released and the air extraction systems may be switched off. Taking into account the complexity of the area and the fact that its remediation goes on by subsequent lots (B2-B6 and C1-C2), INAIL-DIT has requested the verification of the remediation and release of the single restoration certificate at the end of the activities in each lot. It has been also requested to leave at the same time the confinement of the various lots, in order to avoid reciprocal re-contamination processes. At the end of the last remediation, a final inspection by the local control Authority it's requested, for the issue of the final site restorability certificate. INAIL-DIT has also specified that indoor environmental monitoring has to be carried out by sampling at least 3.0001 of air with a flow of 10 1/minute. Finally, it's requested that the visual inspection also should 
cover the most hidden parts of industrial shed. With this aim, an elevation system should be provided to allow the technicians of the control bodies to reach the highest portions of the sheds.

\section{Conclusions}

In the present paper, a review of the history of the Broni asbestos industrial plant is reported, which in the past produced Asbestos Containing Materials, together with the remediation activities in progress. The various project proposals have been examined and the results of the checks carried out on-site by INAIL-DIT have been reported. The critical issues have been identified and the design changes requested by the Institute have been highlighted, in order to protect the workers safety and the neighbouring living environments. The prescriptions indicated may constitute an international reference tool for the design and management of similar remediation activities to be carried out in plants contaminated by asbestos. This paper also highlights the importance of a technical consultation of the national research Institutes before the interventions, with the aim to a design review of the remediation project. These Institutes, superior to local Authorities, own in fact greater scientific expertise on a national and international scale, so they can therefore have an overview of the best available technologies to be applied at sustainable costs, showing also a totally independent judgment, without local conditioning.

\section{References}

[1] F. Paglietti et al., "Guidelines for asbestos remediation at Italian Superfund Sites," Journal of Environmental Science and Health, Part C: Environmental Carcinogenesis and Ecotoxicology Reviews, vol. 30, no. 3, pp. 253-286, 2012.

[2] D. Marsili et al. "Asbestos Ban in Italy: A Major Milestone, Not the Final Cut International," Journal of Environmental Research and Public Health, vol. 14, no. 11, p. 1379, 2017.

[3] E. K. Silbergeld, "Managing hazards in place: The risks of residual risks," Environmental Research, vol. 158, pp. 806$811,2017$.

[4] E. Krakowiak et al., "Environmental exposure to airborne asbestos fibres in a highly urbanized city," Ann Agric Environ Med, vol. 16, pp. 121-128, 2009.

[5] S. Malinconico, F. Paglietti, B. Conestabile Della Staffa, S. Bellagamba, P. De Simone, "Asbestos landfills: Italian data and waste management at support," 31st International Conference on Solid Waste Tecnology and Management, Philadelphia, USA, 2016.

[6] D. Spasiano and F. Pirozzi, "Treatments of asbestos containing wastes (Review)," Journal of Environmental Management, vol. 204, pp. 82-91, 2017.

[7] A. F. Oberta et al., "Asbestos Control: Surveys, Assessment, Abatement, and Maintenance," ASTM International, 3rd ed, 2015.

[8] F. Paglietti, S. Malinconico, B. Conestabile Della Staffa, S. Bellagamba, P. De Simone, "Classification and management of asbestos-containing waste: European legislation and the Italian experience," Waste Management, vol. 50 pp. 130-150, 2016.

[9] J. Wroble, T. Frederick, A. Frame, D. Vallero, "Comparison of soil sampling and analytical methods for asbestos at the Sumas Mountain Asbestos Site - Working towards a toolbox for better assessment," Plos One, vol. 12, no. 7, p. e0180210, 2017. 